\title{
Design of long-distance transmission circuit of high precision micro-seismic signals for mines
}

\author{
Hua Zhang ${ }^{1, a}$, Binxin $\mathrm{Hu}^{1, \mathrm{~b}}$, Tao Jiang ${ }^{2}$, Shaowei $\mathrm{Ge}^{2}$, Feng Zhu ${ }^{1, \mathrm{c}}$, and \\ Tongyu Liu, ${ }^{1, d}$ \\ ${ }^{1}$ Laser Research Institute, Qilu University of Technology (Shandong Academy of Sciences), Jinan, \\ 250014, China \\ ${ }^{2}$ Jinan Power Supply Company of Shandong Electric Power Corporation of State Grid, Jinan, \\ 250001, China \\ ahua.zhang@sdlaser.cn, bbinxin.hu@sdlaser.cn, ${ }^{\text {ffeng.zhu@sdlaser.cn, }}$ \\ tongyu.liu@vip.iss-ms.com
}

Keywords: long-distance transmission circuit; high precision; isolation technology.

\begin{abstract}
This paper presents a long distance transmission circuit of high precision micro-seismic signals for mines. This circuit implements current loop and isolation gate to improve the precision of mine micro-seismic signal acquisition and data quality. The test result shows that the transmission circuit can transmit micro-seismic signal for $10 \mathrm{~km}$ in real time and the dynamic range of the circuit is $77 \mathrm{~dB}$. The circuit demonstrates low power consumption and high precision capability.
\end{abstract}

\section{Introduction}

High-sensitivity micro-seismic geophones is usually buried in harsh environment such as open-pit mines and underground mines, which can locate micro-seismic events quickly and effectively, so it is important for mine safety to do monitor, forecast, and early warning work well [1]. Once the micro-seismic events happened, micro-seismic geophones will generate voltage signals as low as a few microvolts and as high as dozens of volts, then the voltage signals will be transmitted into data acquisition device, as shown in Figure 1 [2] .

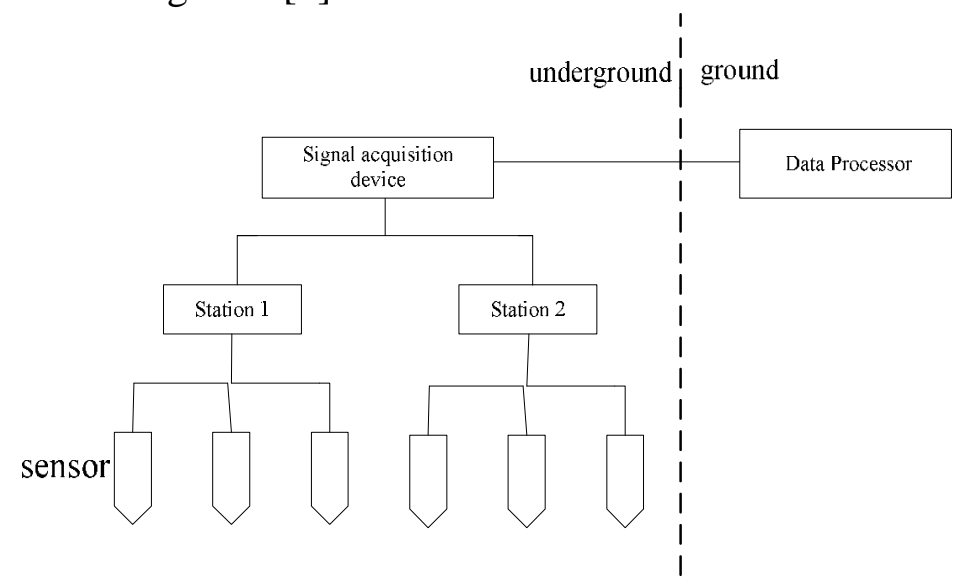

Figure 1.Seismic monitoring in mines

But the length of cable which can transmit the signal from the downhole to the ground is more than $10 \mathrm{~km}$, and the impedance is very large, so it may results in voltage signal attenuation. ${ }^{[3]}$ In addition, the electromagnetic interference in the coal mine is more serious ${ }^{[4]}$, and further it can causes the test signal severely deviates from the original signal, eventually, the analysis result can't reflect the micro-seismic events truly. Therefore, it is very important to design high-precision circuits which can transmit micro-seismic signal in long distance.

This paper proposes a high precision long-distance transmission circuit, capable of transmitting micro-seismic signals for $10 \mathrm{~km}$ in real time and without distortion. It can improve the reliability of the micro-seismic monitoring, and reduce the installation cost and maintenance cost of the equipment. 


\section{Circuit Design}

The designed circuit consists of a signal sending circuit, cable, and signal receiving circuit, as shown in Figure 2. The signal sending circuit converts the voltage signal detected by micro-seismic geophones into current loop in order to reducing signal attenuation in the cable, then the signal is transmitted by the cable into signal receiving circuit. The signal receiving circuit can convert the current loop signal into voltage signal with adopting electromagnetic isolation method, at last the signal is transmitted into ground acquisition device.

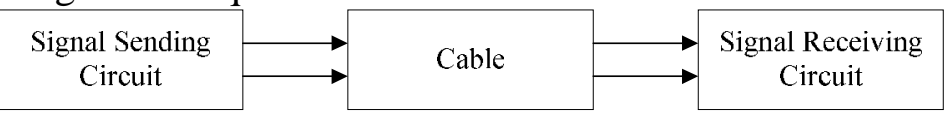

Figure 2. The architecture of long-distance transmission circuit

\section{Design of signal sending circuit}

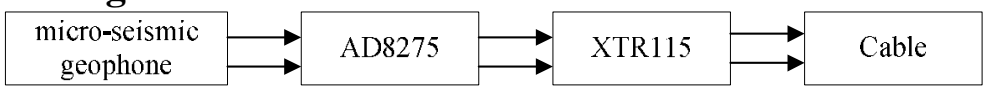

Figure 3. The signal sending circuit

In Figure 3, the AD8275 is a differential amplifier with a gain of 0.2, which converts the voltage of $\pm 10 \mathrm{~V}$ to $0 \sim 4 \mathrm{~V}$. XTR 115 is a two-line current transmitter that converts the voltage signal generated by the micro-seismic into the current signal through resistance, in the meantime, the signal is magnified 100 times. The output signal is the standard current loop signal of $4-20 \mathrm{~mA}$. When the current loop signal is transmitted in the cable, it can reduce the noise and electromagnetic interference, and reduce the voltage drop caused by the resistance in the cable, and improve the high fidelity of the micro-seismic signal transmission. The voltage signal detected by the micro-seismic geophone is amplified by the AD8275 and the XTR115 can convert the signal into current loop, then the signal is transmitted into the signal receiving circuit through the cable.

\section{Design of signal receiving circuit}

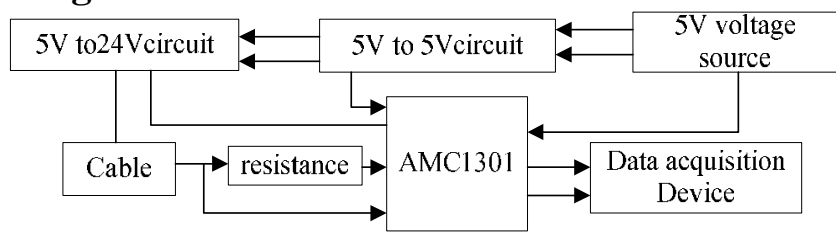

Figure 4. The signal receiving circuit

As shown in the Figure 4, the current loop is converted into the voltage through resistance and as the input signal of AMC1301. The signal receiving circuit is powered by a $5 \mathrm{~V}$ voltage source, and the $5 \mathrm{~V}$ to $24 \mathrm{~V}$ circuit module provides voltage for the signal sending circuit. AMC1301 has an isolation gate with high anti-magnetic interference, and has an amplifying function, with a fixed gain of 8.2. AMC1301 can prevent the noise current in the cable transmission line from entering the signal receiving circuit and improve the signal acquisition accuracy. It can also accurately control the current, reduce the system power consumption and reduce the torque ripple.

\section{The test of the long distance transmission circuit}

Adopting long distance simulation cable circuit, we test the long distance circuit. The resistance of the simulation circuit is $6.2 \Omega$ per kilometer, in the same way, the capacitance is $68 \mathrm{nF}$, and inductance is $470 \mathrm{uH}$. The test system is as shown in the Figure 5.

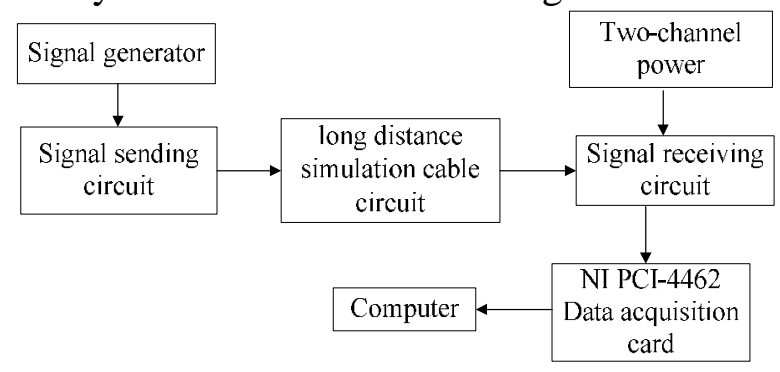

Figure 5. The test system 
The signal generator simulates the signal of micro-seismic geophone, which is used as the input signal of signal sending circuit, and the two-channel power supply 5Vvoltage for the system. Finally the computer collects data by the NI PCI-4462 data acquisition card, as shown in the Figure 6.

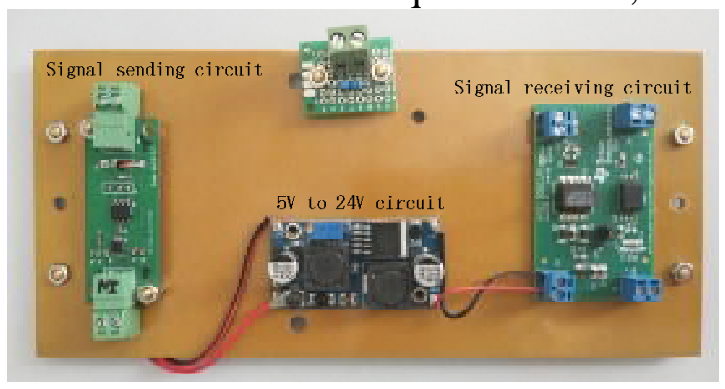

Figure 6. Experiment test

Firstly, the static test of the long-distance transmission circuit is carried out. The output signal was observed by simulating the input signal of $\pm 10 \mathrm{~V}$ DC power, as shown in Table 1 .

Table 1. DC power input test

\begin{tabular}{c|c|c|c|c|c|c|c|c|c}
\hline $\begin{array}{c}\text { Input } \\
\text { voltage(V) }\end{array}$ & 0 & 2.5 & 5 & 7.5 & 10 & -10 & -7.5 & -5 & -2.5 \\
\hline $\begin{array}{c}\text { Output } \\
\text { voltage(V) }\end{array}$ & 1.011 & 1.212 & 1.413 & 1.614 & 1.823 & 0.204 & 0.406 & 0.607 & 0.809 \\
\hline
\end{tabular}

Table 1 shows that the circuit can work normally, and the sending circuit can normally transmit 4-20mA current loop, and the receiving circuit will convert current loop signal into voltage signal.

Secondly, the dynamic test of long-distance transmission circuit is carried out. When the input signal is $0 \mathrm{~V}$, the result is shown in Figure 7. It can be seen from Figure 7 that the interference voltage of the long-distance transmission circuit is $26 \mu \mathrm{V}$. When the input signal is sinusoidal signal with the amplitude of $10 \mathrm{~V}$ and a frequency of $1 \mathrm{kHz}$, its output voltage is shown in figure 8 . It can be calculated from figure 7 and figure 8 that the dynamic range of the circuit is $77 \mathrm{~dB}$.

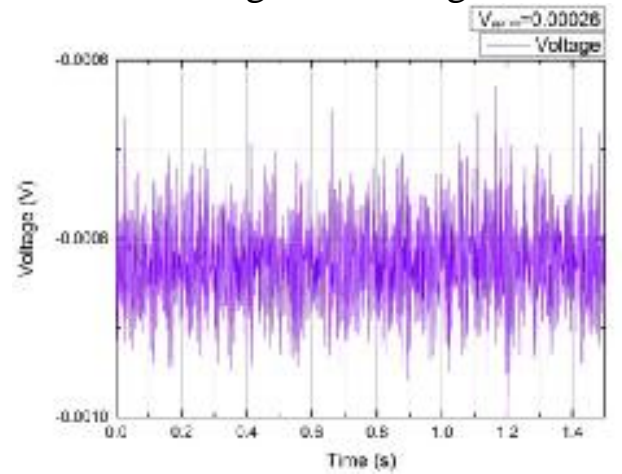

Figure 7. 0V input test

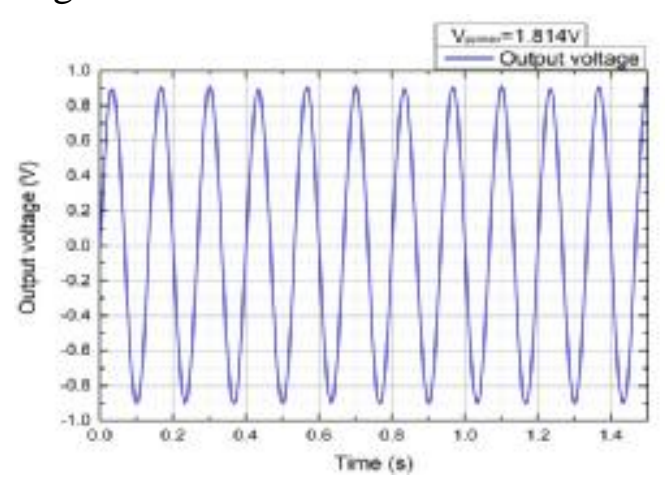

Figure 8. $10 \mathrm{~V}$ input test

Micro-seismic signal transmission circuit is respectively connected $2 \mathrm{~km}, 5 \mathrm{~km}$ and $8 \mathrm{~km}, 10 \mathrm{~km}$, $15 \mathrm{~km}, 16$ kilometers, 17 kilometers, $18 \mathrm{~km}$ to simulate cable, and do the test. The data is done the normalization processing, frequency domain characteristic curve is shown in Figure 9 and Figure 10.

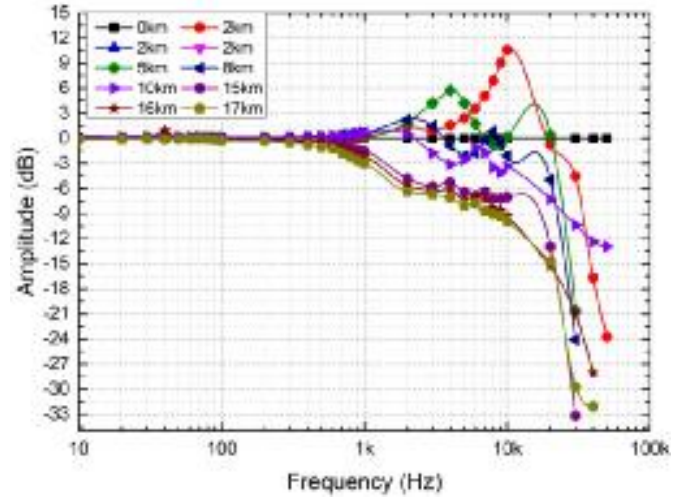

Figure 9. Amplitude frequency curve

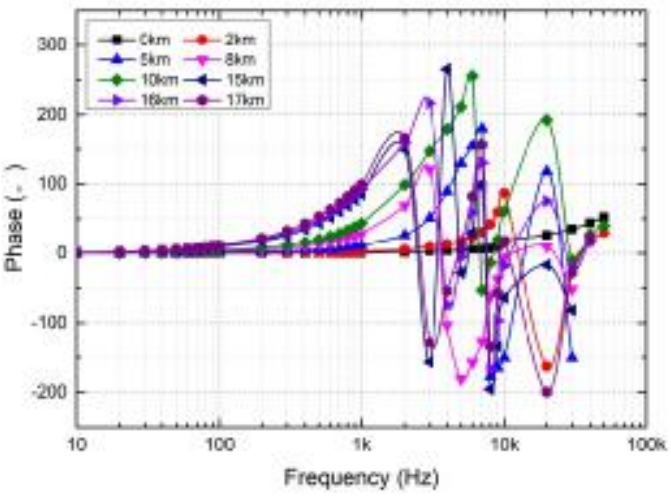

Figure 10. Phase frequency curve 
In the Figure9, when frequency is more than $10 \mathrm{kHz}$, the output voltage signal is rapidly falling down, and the signal transmission distance is $10 \mathrm{~km}$; in the case of low frequency, with the increase of signal transmission distance, the signal can transmitted with almost no signal attenuation and distortion. Due to the low frequency of the micro-seismic geophone, when the signal is not distorted, or equivalently the amplitude is less than $-3 \mathrm{db}$, the maximum distance of signal transmission is $16 \mathrm{~km}$. As shown in the figure 10, when the frequency is less than $2 \mathrm{kHz}$, phase increases with the increase of signal transmission distance; when the frequency is greater than $2 \mathrm{kHz}$, with the increase of frequency, phase decrease firstly and then increase. In the same frequency, with the increase of signal transmission distance, the phase value is increasing. Therefore, in the working frequency range of the micro-seismic geophone, the long distance transmission circuit can transmit the downhole signal to the ground without distortion in real time.

\section{Conclusions}

This paper describes the high precision long-distance transmission circuit for mining adopting isolation gate with high anti-magnetic interference. The circuit eliminates electromagnetic interference of the signal transmission, and it enhanced the applicability of the circuit in the coal mine environment. A large range of tens of $\mathrm{kHz}$ bandwidth dynamic seismic signal accurate conversion and more than $10 \mathrm{~km}$ distance transmission without distortion is achieved. It improved the precision of mine micro-seismic signal acquisition and data quality. The circuit is intrinsically safe circuit, and it has advantage of low power consumption, small volume and high reliability, however its cost is low and it's easy to install and use.

\section{Acknowledgment}

This work was partly supported by the Technology Project of the State Grid Corporation (Research and Application of Key Technology in Intelligent Cable Tunnel) and the Key Research and Development Plan of Shandong Province (No. 2018GSF120008).

\section{References}

[1] Zhang F X, XueBing WU, Shujuan LI, et al. Fiber Laser micro-seismic geophone Research and Application Prospects[J]. Progress in Geophysics, 2014.

[2] ZhenDong, QingtianDong, et al. Research on velocity and acceleration geophones and their acquired information[J]. Applied Geophysics, 2012, Vol.9 (2): 149-158 .

[3] Wang H D. Design and Application of Long-distance Transmission of Sensor Signal[J]. Electric Explosion Protection, 2014.

[4] Wang J, Hu B, Li W, et al. Design and application of fiber Bragg grating (FBG) geophone for higher sensitivity and wider frequency range[J]. Measurement, 2016, 79:228-235. 